\title{
Plug-in Electric Vehicle to Cloud Data Analytics for Charging Management
}

\author{
Md. Muzakkir Hussain ${ }^{\# 1}$, Mohammad Saad Alam ${ }^{* 2}$, M.M. Sufyan Beg ${ }^{\# 3}$ \\ ${ }^{*}$ Department of Computer Engineering, Aligarh Muslim University, UP, INDIA \\ * Department of Electrical Engineering, Aligarh Muslim University, UP, INDIA
}

\begin{abstract}
Electric, plug in electric and plug in hybrid electric vehicles (xEVs) are receiving an utmost attention from automobile industries, policymakers, $R \& D$ agencies and vehicle vendors in the contemporary smart transportation era. Penetration of electric vehicle fleet into the existing charging infrastructure multiplies the load on the underlying grid system. Smart grid technologies in collaboration with smart charging management strategies may circumvent the power load, thus enabling a reliable, efficient, consistent and flexible operation of the underlying power grid system. This work demonstrates commercially viable multi-tier cloud enabled vehicle to cloud (V2C) smart fleet charging model for coordinating the charging of xEVs fleet that can support vehicle mobility satisfying the triangle equivalence namely minimum charging tariff, shortest travelling distance and minimum waiting time at the charging station. Following a multi-tier cloud infrastructure for interactive and real-time control and monitoring of the massive vehicle fleet, this work also highlights the Big Data research thrusts, opportunities and challenges that are being evolved due to integration of a distributed cloud framework with the intelligent entities like smart meters, smart charging stations, $x E V s$ etc. for commercial viability, implementation and deployment of emerging transport oriented cities (TOCs).
\end{abstract}

Keyword- Big-Data, Range anxiety, Vehicle to Cloud, xEVs Charging Management

\section{INTRODUCTION}

The development agenda in smart cities primarily demand green technology transportation. Proactive measures are needed to nullify the gaseous emissions from vehicles run by non-conventional fuels like gasoline [1]. Due to the substantial price-hike in the crude oil and prolonged dependence on foreign oil over the past decades, power GENCOs as well as the consumer, are directly or indirectly forced to opt for alternative energy sources [1]. The hybrid cars introduced by automotive industries like Nissan, Honda and Toyota are a step towards this innovation attempting to replace the Internal Combustion Engines (ICEs) with rechargeable batteries and electric motors. The bulk of the research is going on to ensure a zero-emission mode of transport. Fortunately positive results have been shown by such inventions as Electric/Plug-in electric/Plug-in Hybrid Electric Vehicles (xEVs) which can serve as the best recourse in this storyline. Conceptually, xEVs integrate the electrical networks with so called data and communication infrastructures through smart metering and sensing utilities [2]. The heterogeneous data generated from these devices roots the use of efficient data management techniques to deliver a consistent, reliable and real time operation of the massive vehicle fleet [3]. Cloud computing is an emerging approach, that is envisaged to provide a flexible, secure and cost effective platform for storage, as well as execution of the computing resources thereby offering a robust architecture destined to have a reliable and real time operation of engaged utilities [4]. Coupling the utilities with the cloud services ensure a naïve user to gain an anytime and anywhere access to the network as well as shared resources through smart applications at his disposal [5], [6]. Moreover, the massive penetration of utilities into the cloud infrastructure triggers tsunami of data generation synonymously "Big Data" [4], [7]. Robust data analytic tools and techniques supplemented with fast processing elements are required to manage and mine such datasets, with an aim to come up with a smart decision making paradigm [6], [7], [8].

\subsection{MOTIVATION}

With the advent of rigorous research and development efforts and stringent protocols related to vehicle emissions [9], fuel economy, constraints in conventional energy reserves and so called global warming, electric vehicles specifically electric/plug-in electric and plug in hybrid electric vehicles (xEVs) are receiving an utmost attention from automobile industries, governments, R\&Ds, vehicle vendors as well as consumers as they seem to serve as the sustainable and efficient power trains for the coming generations. According to survey in [10] and [11], during a short period of six years from 2008 to mid of 2014, about a quarter million plug-in hybrid electric vehicles have already been launched into US roadways. As of 2013, more than 129,500 Americans were driving plug-in EVs manufactured by all major automotive OEMs [12], while the registration process is still on the pace in countries like France, Germany etc. The tremendous increase in the xEVs population has created an alluring interest in the research community to invest their efforts in management of such vehicle fleet. A study in [13]-[15] reveals that perforation of large scale xEVs fleet can pose a big challenge as they can disrupt the underlying grid network unless their behaviors are monitored and coordinated properly. The side effects may be 
in the form of power losses, incremental investment on the pre-existing network, potential violations of statutory voltage limits, degradation in power quality etc. Lack of coordinated charging strategies can also create demand peaks during rush hours which in turn put pressure on the power grids [11]. Above all, the utmost concern is the "RANGE ANXIETY" felt by drivers of conventional vehicles. In a nutshell, while executing a fully electrified fleet, the uncoordinated charging of xEVs population may pose serious impact on reliable and efficient operation of the associated supply system [9], [11], [16]. Use of proper charging strategies may circumvent significant proportion of burdens from the overall architecture [11]. Indeed, it has been empirically estimated that even if all the vehicles on the road are made to run by electricity, the existing power backbone can withstand the hike in power demand provided that they are intelligently managed [17]. However, coordination of such electric vehicle fleet can only be fulfilled through deployment of smart transport architecture equipped with intelligent agents like smart vehicles, advanced metering utilities and sensors etc. as shown in Fig.1

But congregating these smart members into a single dais and further administering them is a bewildering task as galactic volume of data is generated in the course of installation of the framework that employs such entities.

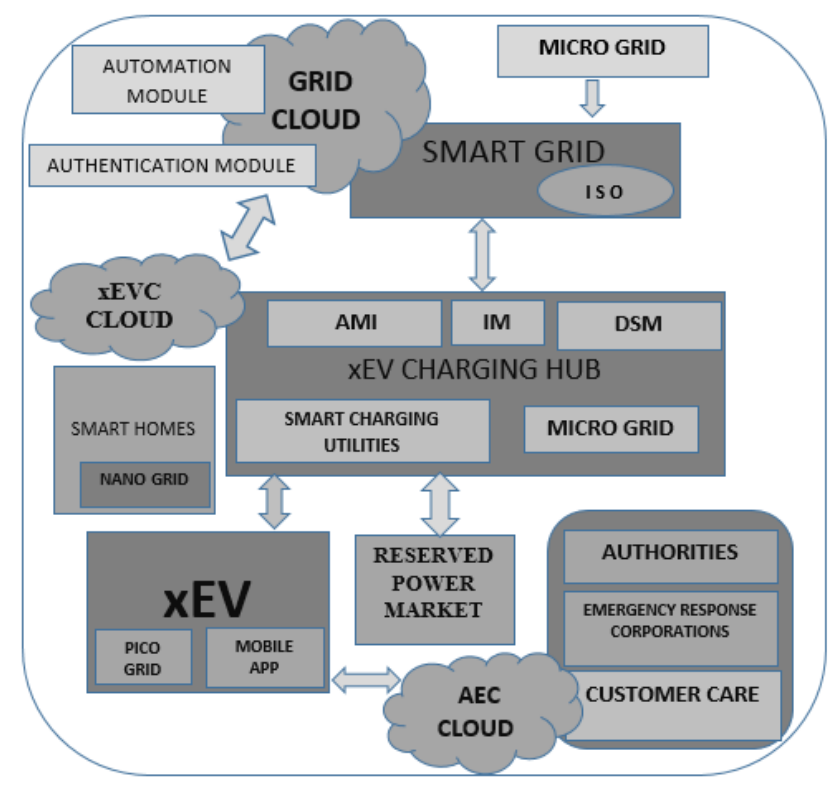

Fig.1. Proposed Architecture of Cloud aware Transport Oriented City

As estimation discovers, the transactions of only two million smart metering populations generate more than 20 GB of data every day [4], the real-time usage data size could certainly manifold. Trends reveal that the vast scale deployment of smart transport system leveraged with Advance Metering Infrastructures (AMI), smart grid, smart charging stations as well as intelligent xEVs is still in its infancy as the state of art management strategies break down to supervise such elements. Installation of such architecture requires robust and efficient analytics framework for gathering, storing, processing and managing the data originated from the intelligent utilities. Hiring cloud services is envisioned to play vital role of motivation in devising a storage and execution setting for the aforesaid components. The cloud can be simulated to act as cache for the generated data. The usage data in smart meters and sensors can be exploited to discover useful patterns, which may be learned to design predictive algorithms and thus building an efficient decision making environment. The operations of the entities like xEVs can be tracked through remote cloud in real time thereby enabling strong and consistent governance for the whole infrastructure.

Unfortunately, coupling the cloud into the prevalent infrastructure triggers exponential breeding of data further magnifying the exertion on the storage and processing elements. The integration of cloud computing with smart transportation electrification and energy management further manifolds the heterogeneous data generation. The need for scalable, fast and high performance computing approaches, such as distributed or parallel computing, is strongly perceived in such settings. Indeed, the nature of fast, dynamic and time series data welcomes scalable big data processing technologies that can withstand and learn from such evolving networks. The size of generated datasets grows exponentially with the counts of utilities as well as consumers, giving birth to precise and accelerating data challenge for the data analysts. The gigantic and complex nature of the smart grid network compels researchers to use robust graph analytics to harness the node structure of the utilities and consumer distribution that may aid to establish an intelligent and self-learning configuration. Driven by the issues mentioned above, there is resurgence of research interest in the data analytics community to incorporate cloud computing paradigm leveraged with graph based big data analysis algorithms into the smart 
xEVs charging infrastructures as the future of sustainability for the $\mathrm{xEVs}$ lies on the way they are being used and coordinated in the coming days.

\subsection{CONTRIBUTION}

There has been a consensus acquiescence in the industries as well as research communities on the fact that an intelligent and duplex trading of data and control between the plug in hybrid electric vehicles and recharging sockets through the use of smart meters and other such amenities would empower in prototyping strong decision making paradigms. Furthermore, recent studies and trends prompt researchers to incorporate intelligence in the charging infrastructure. The intention of this research is to provide a commercially viable model, ready to be prototyped as described in the patent filed with name V2C [18]. It also incorporates cloud data analytics into the infrastructure claimed by proposing a smart V2C remote charging infrastructure for the electric/plug-in electric/plug-in hybrid electric vehicles (xEVs) as shown in Fig.1. In this framework, the charging methodologies and decisions will be coordinated through remote processing and analytics agents employed at the cloud data centers in a master-slave paradigm. The involvement of cloud into the management manifesto deemed to ensure a consistent, reliable, sustainable and cost effective execution of the xEVs vehicle fleet. A three-tier cloud architecture is formulated that explores the state of charge (SOC) of the xEVs and parameterizes the different levels of range anxieties in the vehicle driver and correspondingly supplements incredible solutions aiming to assure the xEV driver a range anxiety free drive. Simultaneously, the cloud services are leveraged to track the state of reserve (SOR) status of both smart charging stations as well as the back-end grid network, and allowed to switch to alternate modes of demand response via micro and nano-grids. Several mining strategies specifically kernel based graph analytics strategies are also addressed that could harness and infer from the catastrophe of dynamic data and establish a self-learning test bed that can evolve accordingly. The contributions can be encapsulated as follows-

1. Introduction of the notion of cloud aware transport oriented cities (TOC), comprising of intelligent entities like Smart grid, Smart charging station, Smart car and Smart meter.

2. Presentation of a commercially viable realization of vehicle to cloud (V2C) framework in the TOCs destined to smart charging management of forthcoming smart xEVs fleet satisfying three criteria namely Minimum charging tariff, Shortest travelling distance and Minimum waiting time.

3. Proposal of a control flow model for Big-Data to Knowledge (B2K) framework, for efficiently harnessing the data that may generate in the installation of the cloud controlled transport infrastructure.

This manuscript is organized as follows. In section 2, the architecture of the Cloud Enabled Transport Oriented City is proposed with highlights of the prevalent active researches along the domain. Section 3 describes the proposed $V 2 C$ model in detail. Section 4 proposes a big data processing framework that mines huge datasets from cloud data centers, sensors and other data sources. Conclusions and future prospects are provided in section 5 .

\section{BACKGROUND}

Recently the installation smart city is envisioned to constitute a global research endeavor [9]. Policymakers as well as R\&Ds have joined the consortium that is channeling its academic and research expertise towards the deployment of transport oriented cities (TOC). These cities are uniquely distinguished by provisions for intelligent data connectivity. Researchers require comprehensive knowledge of power consumption trends, user habits, resource usability and availability in order to induce such smart environments. There exist numerous proposals about how to benchmark the features of a smart city but it is a strong hope that transport framework emblazoned in this work could achieve a worldwide consensus emphasis. The prime ideology on which the model is established is remote accessibility and remote management. Plenty of claims and proposals are found in the literatures which are working independently in diverse domains such as security in information management of smart grids [4], smart grid dynamic energy management (DEM) [8], agent based simulation of electric vehicles fleet charging strategies [9] and many more. Fig. 1 depicts the notion of TOC infrastructure comprising of four SMART's namely Smart grids, Smart charging stations, Smart cars and Smart meters.

As the specifications of smart grids are in a state of persistent evolution, plenty of literatures are found competing to settle consensus taxonomy. However U.S. Department of Energy (DOE) Office of Electricity Delivery \& Energy Reliability (OE), describes a smart grid as the one having seven prime functionalities: Integrating all generation and storage means, customer participation, new markets and operations, self-healing from disturbances, power quality options for the twenty first century, asset optimization and operational efficiency and resiliency against attacks and disasters [19]. According to the same report, by 2030 DOE plans to furnish power grids into a smart power delivery system equipped with plug and play integration of sporadic and dispatchable low carbon energy sources, establish a consumer interactive platform for load management, international fuel independence, innovation, business model and modules to ensure economic privacy and security [17], [19]. A smart charging station is interfaced to smart grid, smart vehicle as well as smart meters. It may have intelligent charging mechanisms to learn from the state of charge (SOC) of the plug in hybrid vehicles 
in the vicinity as well as the level of range anxiety in the vehicle driver and correspondingly schedule the charging procedure in such a way that the peak load on the back-end grid systems is properly shaped. The consumers can actively participate in the demand response events and the stations have modules that wield usage patterns to avail vehicle specific tariffs or recommendation to the user [8], [16]. Further, a smart charging station may be equipped to have efficient charge scheduling strategies and pricing mechanisms, such that it never remains idle.

Hypothetically, a smart car is an autonomous or synonymously a driverless car, an automobile capable of learning and driving itself, in that the control and enhancement operation are automated. Smart cars are vehicles leveraged with system-driven forms of artificial intelligence; they have their own operating systems. Proponents claim that the widespread use of smart vehicles equipped with decision-making modules would help to achieve much safer roadways than today's traditional counterparts. Though the idea of this technological innovation has been around for decades, the reality is not quite as advanced as theatrical portrayals and there continues plethora of research activities in testing and deployment of smart car innovations. A smart car seems to be a panacea for next generation transport, being more efficient than their combustion engine counterparts. Electric/Plug-in \& Plug-in hybrid electric vehicles (xEVs) best fit the smart car definition as they are fuel efficient, zero emissive and made to run through renewable based power alternatives [1]. A smart car may have support for vehicle-togrid (V2G) power and control flow that enables it to participate in the dynamic demand response and load management system, either by vending the electricity or by choking its charging rate [16]. Smart electricity meters offered with intelligent functionalities are strong prospect for future XEVs transport [8], [19]. Smart metering software installed on handsets can use the cellular signals for having multi-way communication of data and control among the entities like vehicle driver, actors at charging stations, smart grids, authorities and many more [3]. Indeed, a smart meter may have their own databases that can assist intelligent mining procedures motivated to provide more insightful knowledge helpful in adopting smart decision making and optimization algorithms. Smart meters deliver a transparent and real-time environment for consumer services such as energy billings, SOC monitoring and remote location tracking [3], [20]. As in our case, smart metering may be employed at multiple interfaces such as xEVs and cloud, xEVs and smart grid, xEVs and charging station and charging station versus smart grid. By executing proper synchronization mechanism at vehicle-grid interface, smart meters can have automatic outage detection mechanisms for sending continuous feedback. Competing research is going on both in industries as well as academics, to program various modules for dynamic data collection, performance management system and use them as tools for data analysis, prediction and recommendation and reporting [6], [8].

Cloud computing synonymously on-demand/on-lease/pay per use computing, is an internet based flexible, cost effective, scalable and data and energy efficient computation approach that has ingeniously renovated the traditional computation approaches [4]. Agility, virtualization, independency of device as well as geography, maintainability, productivity, reliability, scalability and elasticity are notable features that make it a potential computing model for contemporary business and research in large-scale and complex computing [5], [8]. The so called on demand computing paradigm seems to manifest as multi-tenant in accomplishing complex tasks required to mine the giant datasets, thereby eliminating the use of expensive hardware and also ensuring an optimum time and space complexity. Cloud computing paradigm best fits the proposed scenario where proper synchronization mechanisms could be installed to control and monitor the smooth working of the acting entities

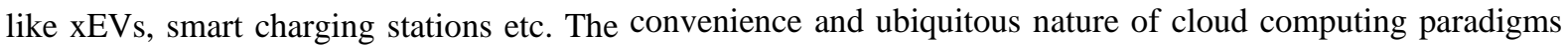
has paved the way for cloud computing to new heights and constitutes one of the most significant shifts in modern Information \& Communication Technology (ICT) [4].

The rising trends in wireless communication and ubiquitous smart devices led to emergence of mobile cloud computing applications like such as Gmail/Google Drive, iCloud, and Dropbox, etc. which act as outsourcing bed for user task and allows its customers seamless access to services from anywhere along the globe. There exist varying levels of services offered by cloud computation like PaaS, SaaS, and IaaS [4]. Saas constitutes the topmost layer for the cloud computing architecture where the services are delivered in broadcast mode, i.e. the consumer gains public access to the service installed in the remote cloud hardware [9]. Multiple users are allowed to have the services via APIs or web browsers at the same time only after appropriate authentication constraints are fulfilled. Popular examples of SaaS servicing agents are Google Docs, Gmail, Salesforce.com, Google power meter, etc. IaaS frames the backbone of the cloud system. It refers to infrastructure equipment like Virtual machines, servers, storage and network etc. for operating on a cloud and are offered as plug-in tools by cloud vendors to the consumers and other utilities. Through virtualization, IaaS reinforces computational and storage capabilities and enforces load balancing protocols to run applications in diverse domains like smart grids (SG) that demand high performance. Modules related to information management tasks like gathering, storing and processing, etc. all are implemented in this mode. Flexiscale and Amazon's EC2, GoGrid, 3Tera etc. are examples of prevalent IaaS services. Platform as a Service $(\mathrm{PaaS})$ is responsible for offering the operating environment i.e. database, deployment tools and web servers, etc. needed to develop runtime environment. It 
delivers tools and libraries and also decides the programming models to develop higher levels of cloud computing services. Google's App Engine, Azure service platform, Force.com are notable examples of these type of services.

Moreover, there may be provisions for numerous service models that can be encapsulated as XaaS where $\mathrm{X}$ stands for Anything and it may be data, network, identity, strategy, business etc. giving rise to DaaS, NaaS, iaaS, saaS and BaaS respectively. There are multitudes of deployment models for cloud computing specific to desired level of granularity. It may be Public Cloud, Private Cloud, Community Cloud and Hybrid Cloud. The Public cloud exposes the services for public access. Private Cloud as the name suggests, is an internal deployment paradigm which is designed exclusively for personal enterprise; obviously confidentiality, privacy and security is its prime concern [4]. In Community Cloud counterparts of the domain of Private Cloud are unlocked to organizations or groups of users only. Hybrid supports both private as well as public mode of access, depending on the nature of services (whether critical or passive) to be employed [5], [7]. In a Hybrid Cloud, inter-cloud communication may be leveraged; enabling to hire services from third party Cloud Providers and thus it increases the flexibility of computing. Private Cloud services may be augmented by collaborating the tasks and resources to and from Public Cloud and which may be utilized to manage any unexpected surges in workload [5]. For the proposed work, the Global Positioning System (GPS) Cloud performs the role of Community Cloud with which the $\mathrm{xEV}$ drivers communicate, while charging stations and smart grids employ Private Cloud for their purpose. The authorities, emergency response corporation (ERC) and customer care services are also interfaced to the xEV application via Hybrid Cloud. Fig.2 shows the interaction among corresponding clouds at varying deployment levels.

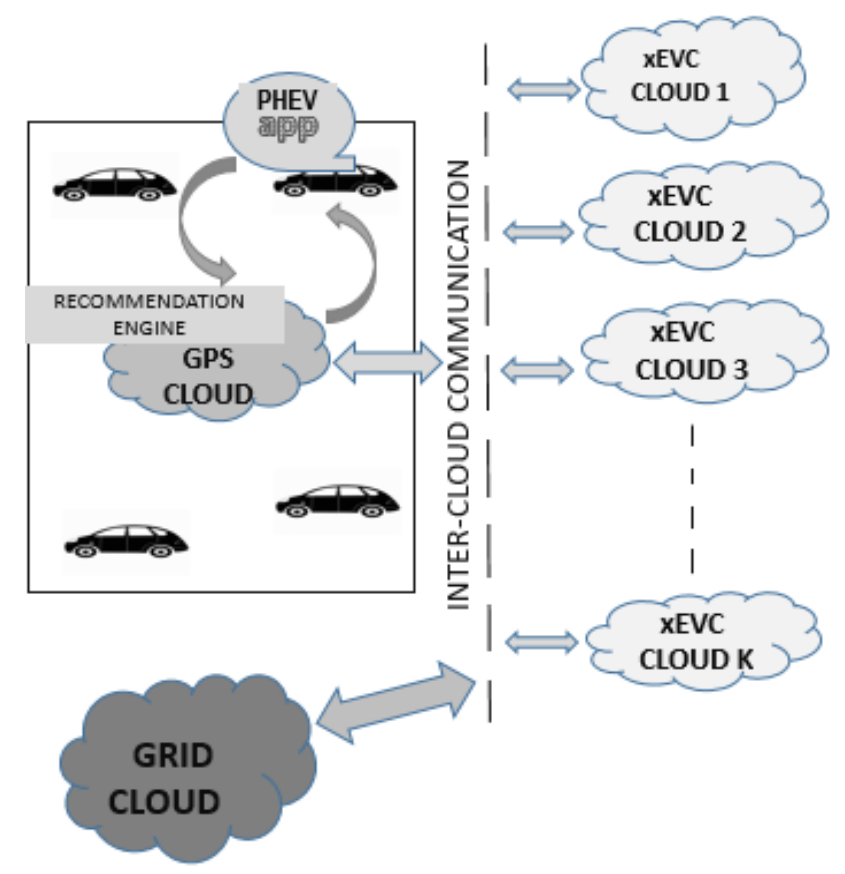

Fig. 2. Block diagram of interaction strategies of various cloud deployment models

\subsection{RELATED WORK}

There persists a full swing of research in adopting the best practices to manage intercity and intracity electric/plug-in and plug-in hybrid electric vehicle (xEVs) charging mechanisms in real time [5], with provisions for vehicle-to-grid (V2G) technologies [20], to achieve a reliable and dynamic load management and response to demand in the smart grid infrastructure[8], [21]. Many cloud computing model for managing big data in the smart grid have been hypothesized, [4], [5], [7] but they lack an analytical framework supporting inclusion and coordination of xEVs fleet and its data analysis. Authors in [8] addressed many intelligent algorithms for big data management in smart grids and smart meters but it demands implementation support for its feasibility. Designing a globally optimal scheduling scheme is impractical because future information about the arrival of vehicles is not available; consequently is hard to estimate the upcoming load. However they found hypothesized version of numerous coordination mechanisms based on prediction and simulation of future the hybrid vehicle fleet distribution. In [10], the notion of time coordinated charging (TCC) and power coordinated charging (PCC) is proposed, while authors in [11] attempt to formulate a real-time pricing model based optimization problem for global scheduling of xEVs charging and discharging. 
The same work also proposed a local scheduling optimization problem which is claimed to scale its performance close to that of global counterpart. Request from vehicle or users are processed in [3] and [22] to devise hierarchical aggregator-based hybrid vehicles charging control mechanisms that may lower the variability of demand and distributed power generation through smart charging and discharging in real time. Furthermore, authors in [3] also classified xEVs charging strategies into architecture based as well as control based. The former may be designed either in centralized or decentralized mode while there may be direct or incentive based control in the latter. A game theoretic paradigm is adopted in [23] to supervise the random crowd of vehicle fleet thereby safeguarding xEV's privacy issues in terms of charging schedules. In [24], a fuzzy logic Fuzzy Charging Controller (FCC) is proposed that takes the States of Charge (SOC), the grid parameters represented in the system minimum voltage, and the hourly energy price as input parameters to ensure the system threshold voltage within permissible limits. The interpolated FFT algorithm employed by authors of [25] attempts to devise a cost effective interactive charging strategy which maintains grid stability by minimizing the grid load factor, and ceils the optimal battery charging rate within admissible limits. The work in [26] demonstrates the optimization of charging cost of xEVs fleet through moving window optimization scheme. The authors in [27] adopted a dynamic programming approach to simulate a bidirectional control flow model (V2G also enabled) that coordinates the charging scheme. The rise of so called big data due to cloud services along with current research challenges and issues is also addressed in some documents [4], [6], but literature that delivers a robust solution dedicated to the statistics and analytics of such issue is scarce. The model devised in this work, is hoped to be the first realization framework that conjoins charge scheduling of massive xEVs fleet with cloud infrastructure and simultaneously orient BigData solutions to emerged issues and big-data control flow as shown in Fig. 3.

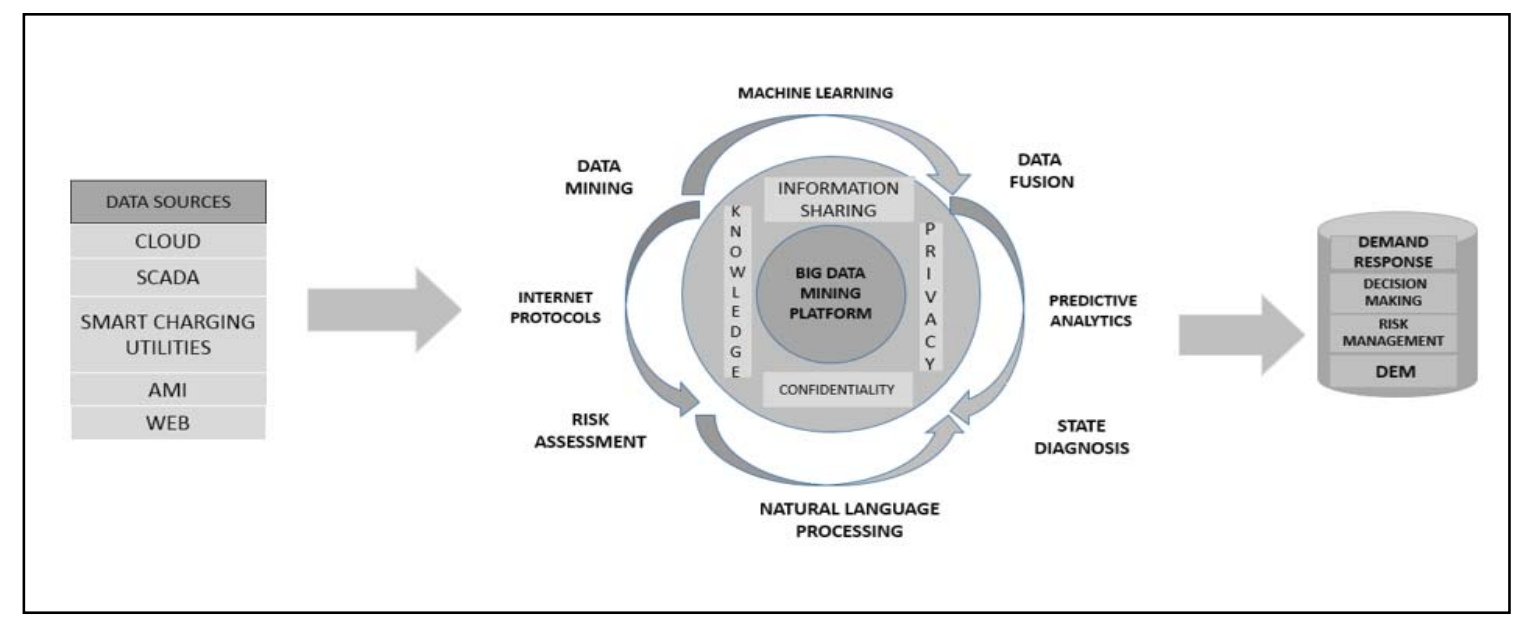

Fig. 3 Control flow model for Big-Data processing in smart grid and Smart Charging Architectures

\section{VEHICLE TO CLOUD (V2C) MODEL}

The proposed model is intended to provide a commercially viable infrastructure ready to be prototyped as described in the patent published in [18] with the name V2C \& simultaneously exploring the challenges that can surface in the interim. The intention is to devise a V2C remote management charging infrastructure devoted to coordinate charging of contemporary $\mathrm{xEV}$ population. The cloud hierarchy and inter-cloud interaction is rendered in Fig. 2. In the proposed V2C scheme the cloud will act as the hub where the entities involved in the infrastructure will have seamless interaction. Obviously, robust computationally optimized algorithms need to be installed on the cloud to ensure the system to trigger necessary controlling operations. The generated data from the metering utilities and sensors will be stored and processed at the data centers. The varying dimensionality in the data entails aggregation analytics to shape the data into proper format. The cloud will represent in three isolated interfaces each with the xEVs, the charging station, and the smart grid. The APIs at the xEVs end needs only to interact with the cloud, and it is not needed to communicate with the charging station and smart grid vendors directly.

There would be a two tier architecture where the charging station and smart grids will engage concerned cloud deployment models on their side and each of these cloud modules will be conjoined to the central cloud that act as hub to monitor the transport for whole city as portrayed in Fig. 1. This requires implementation of efficient and secure communication as well as interfacing procedures 
The API at XEVs end would be interfaced to the hybrid cloud through authentication mechanisms. The hybrid data centers would be under government administration and the hybrid clouds communicate with the former through appropriate authentication mechanisms. Keeping Hybrid Cloud under government administration ensures proper pricing schemes/power rates. At the same time, it also hides the implementation details from casual users by enforcing stringent protocols thereby assuring a secure infrastructure. The centralized control also removes redundancy in the computation.

\section{$3.1 \mathrm{xEV}$ to CLOUD interface}

The application running at xEVs driver Smartphone/PDA will have bidirectional information exchange with the API at Hybrid Cloud to provide real-time updates for the systems at both end. The control signals may be on pricing status, state of charge at xEVs, location of best station, best route/ shortest route, etc. There would also be provision for cloud to the xEVs one way control and tracking signals like tracking signals that are of use to the authorities. The SOC of XEVs will be an indication of levels of "range anxiety" for the driver that can act as the recommendation parameter for the system. The application will track the range anxiety level and correspondingly suggest the driver which station to approach using three modes of recommendation.

A. Minimum Charging tariff

One of the prime objectives of a standard model other than availability is to have accessories and establishment for cheap power. Many a times the consumers are served with an unaffordable charging option that further worsens his concern. This application will monitor the dynamic power pricing at different charging stations and recommend the one with optimum rate. It is discovered from the subsisting charging strategies that the consumers often have to endure the unrealistic price menu due to vendor's monopoly in the market. The distribution of dominion over several dealers would enhance competitiveness among them and so the consumers could grab the edge. The application will also be equipped to surveil the forthcoming pricing agenda and correspondingly prepares the user to manage the trade. Moreover, varying pricing schemes based on intra-brand or inter-brand charge transfers, mode of charging (fast, normal or slow) and charging hours (off peak/ rush hours) may be employed to have a range of services sufficing an array of customers. Obviously, the rate will vary accordingly i.e. fast charging mode is more expensive than charging through normal ports and similarly the price at rush hour will attain the peak.

B. Shortest Travelling Distance

This scenario comes out when the SOC indicates the red level of range anxiety and driver is in fear that his trip may be interrupted in the midway. Obviously the shortest path paradigm needs to be executed for such circumstances. However, it is not guaranteed that the shortest path will always be least time consuming. The contemporary innovation depicts that the use of photoactive coatings on the panes of xEVs may perform the task of nano-grid and thus assures the user to withstand the emergency.

C. Minimum waiting time

Here the driver has time constraints and it generally occurs during office hours. For such case, the road which is shortest as well as having least traffic is the ultimate option. Many a times, the driver has to wait relentlessly. The delay may be in the form of traffic delay, queuing delay at charging station or wait during the charging continues. The on charging delay may be avoided through adopting various modes of charging like fast, moderate and casual, by paying according to the pricing schemes.

\section{D. xEVs Charging Hub/Cloud communication}

The data from the smart charging station equipped with intelligent metering and data acquisition utilities store and process their own computations using the infrastructures like OS, storage elements and databases deployed on the hybrid cloud platform through Infrastructure as a Service (IaaS) services. They also hire the development environment or platform through Platform as a Service (PaaS) servicing strategies and execute their data analytics algorithms. The reserve for charging station is sensed continuously so that power can be replenished before the critical stock, to ensure that no customer in the queue should suffer the limited stock apology. The power drain from the underlying smart grid system should be in a way that there is an even load distribution in the grids. In case of rush hours peak load will be balanced through proper demand response algorithms.

The charging station also employs robust scheduling algorithms, such that the station never remains idle. Each station tracks the xEVs in the vicinity and proposes recommendations to the customer. The recommendation engine installed in the central cloud extracts the individual recommendations from the local cloud, and provides the consumer with a consensus choice. Moreover, the application at the charging station-cloud interface may also track for micro grid alternatives, so as to perform demand response efficiently 


\section{BIG-DATA TO KNOWLEDGE (B2K) FRAMEWORK}

The massive volume of real-time data from the involved entities are stored and processed in the cloud in such a way that an optimized decision making set-up must be established in every interface, thereby maintaining a winwin like strategy at every end of the acting entities. The dynamic and time series data from distinct data sources forms so called Big Data that invoke scalability and flexibility concerns. The large-scale heterogeneous data from the smart metering utilities require BIG DATA management and advanced data analytics. While modeling the proposed scenario, several strategies are adopted which consider different perspectives at each interface. At the smart grid end, the cloud processing technologies adopted should ensure a sustainable, economic plus reliable dynamic energy management (DEM). For that, complex decision making algorithms need to be implemented. The various modules involved in DEM are vehicle-to-grid (V2G) technology, real-time wide-area situational awareness (WASA) for monitoring the grid status, Home Energy management systems (HEMS) for incorporating user's participation and high speed computational tools for supervisory control. The large and multidimensional datasets call for High Performance Computing (HPC) which requires fast and massive storage and computational elements. The significant correlations, patterns and trends in massive datasets obtained from the sensors can be efficiently mined to devise intelligent schemes for demand response and load balancing. Sensors can also serve as the data-source for predictive analytics needed for load forecasting, dynamic pricing, optimal scheduling of resources and bad data correction. These massive datasets can also be exploited through pattern mining, machine learning algorithms or data fusion techniques to predict the load patterns to concoct smart decision making and resource management strategies may be employed. Lack of efficient control and monitoring protocols may escalate the risk of failures due to overloading and congestion. There may be provision for tracking and eliminating faults through use of robust state diagnosis algorithms, thus making the architecture immune to abnormal events and failure. The instabilities and uncertainties in the power market need to be taken into account to devise sound risk management modules for managing the financial risks. Fig. 3 depicts a layered big data processing architecture, along with the control sequence for extracting knowledge and decisions from the data generated in the cloud and smart utilities interaction, which is believed to prove sound in providing intelligent solutions for the profound issues in the postulated infrastructure.

Data mining is an array of methodologies and processes to extract useful knowledge from streams of data. In the context of TOCs, the latter may be xEV user's driving profiles, renewable injection profile, SOC's of xEV, load and demand variation etc. Primary tasks encompassed under data mining are anomaly detection, rule mining (mining associations, patterns, relations etc.), clustering, and classification, summarization and regression analysis. Use of machine learning techniques for TOCs provides the utilities ability to adapt themselves to act, grow and change without explicit stakeholder involvement when exposed to real-time datasets. The data generated in transport oriented architectures demands efficient use of such algorithms as it has significant impact on deciding the decision making processes and system reliability [25]

The entities involved in the infrastructure produces voluminous datasets that need to be processed through dimensionality reduction algorithms to overcome the complexity issues related to storage, analysis. Transforming the original data streams into noble representations through summarization techniques removes the concerns related to scalability, complexity, event detection and process execution. For deployment of efficient charge scheduling strategies short term and very short term demand forecasting methods are to be employed. Data mining and machine learning procedures such as regression based models, expert systems, time series analytics, artificial neural networks (ANN), fuzzy-neural networks, and evolutionary programming etc. had been emerged as computationally potential tools for charging load forecasting. In order to discover groups, patterns and distributions xEV load data, clustering techniques like load classification (LC) can be used. In event-based applications, machine learning algorithms proved to be efficient in devising frameworks to ensure seamless integration of event processing techniques with pre-existing algorithms.

Data fusion (DF) paradigms essentially employ sequence of techniques for aggregating heterogeneous configurations of data from varying sources to acquire intelligence and inferences for the system. Due to the contemporary advancement in transportation and road telematics specifically on board sensors (OBS), localization mechanisms, information storage and processing technologies etc, TOC utilities and data centers are provided with heterogeneous forms of data that require efficient data fusion techniques to reach a comprehensive inference. DF techniques primarily inherit algorithms from three broad domains namely statistics, probability and AI. Statistical approaches include weighted combinations, multivariate analysis and data mining algorithms while Bayesian networks, state-space models, Kalman filter based data fusion techniques come under probabilistic approaches. DF frameworks can also be designed using neural nets, cognitive and genetic algorithms that are incorporated under AI umbrella. The data fusion pipeline as implemented by US Department of Defense (DoD) seems to appear as comprehensive model for the V2C infrastructures, which involves five execution steps with human in the loop. Level 1 involves data preprocessing i.e. compression, normalization and formatting methods etc, the outputs of which are fed to level 2. In level 3, the real-time data obtained from appropriate sources in level 2 are mingled with standard databases to 
trace $\&$ analyze the possible causes for the events occurring in the data. In the next processing level the patterns, correlations and semantics of information are assessed and aggregated. Level 5 extracts the feedbacks from previous steps and applies successive refinement strategies to predict, assess and evaluate the need for further improvement in the DF methodologies. The overall performance is tuned up by involving human factor in the loop to interpret and utilize the output from the DF pipeline.

Though V2C infrastructures in transport oriented cities offer great potentiality for data and energy management, switching from conventional power architectures cloud aware smart grid and xEV charging utilities will introduce risk factors that needs to be carefully mitigated. The multidimensional datasets generated in the V2C model may pose vulnerable threats to the stakeholders, thus necessitates the need to conduct critical risk assessment and analytics. Concerns for maintaining confidentiality, integrity and availability in the transportation and metering utilities have stressed the research folks to work for embedding risk analysis into design in a way that ensures transparency and understandability among involves stakeholders. Though the field of risk management as revealed from literatures suffers from lack of consensus frameworks and ambiguity in risk elements and terminologies, the framework proposed in [29] seems relevant and definitive. It defines the risk management model as consequence of successive phases namely risk scope definition, risk identification, risk characterization, risk evaluation and risk mitigation planning. The outputs of such models are iteratively communicated, reviewed and evolved in course of execution to incorporate resiliency and sustainability in the TOC like infrastructures.

Predictive analytics involves use of statistical, machine learning and data mining techniques to analyze historic and real-time datasets generate rules and predictive models to predict future events. In order to install V2C infrastructures from the scratch and to meet the regulatory constraints for renewables and xEV penetration, utilities require robust predictive planning and analytics that can optimize asset replacement expenses and enhance the execution efficiencies under stringent budgetary ordinances. Through predictive forecasting and asset analytics, softwares and services can be developed to make the utilities in TOC infrastructures aware of the potential events for outages and correspondingly execute workforce planning that can undertake proactive measures for event mitigation and routine maintenance. Predictive asset analytics if intelligently used improves the technological, productivity and business process engagements in a way that assures customer satisfaction, proper route planning, better safety and compliance, and optimized field crews.

\section{CONCLUSION}

In this work the notion of Transport Oriented Cities (TOC) is established that comprises of intelligent entities like smart grid, smart charging station, smart car and smart meter and the interaction among these entities is coordinated through the deployment of a cloud infrastructure. The hierarchical cloud framework optimally control and monitor the components and entities involved in operation of a smart city. A comprehensive and commercially viable realization of vehicle to cloud (V2C) model for smart and coordinated charging of the $\mathrm{xEV}$ fleet is proposed. The cloud data centers are endowed with high performance computational elements and robust data analytics algorithms for delivering a real time solution to achieve smart charging management of smart xEVs fleet supporting three criteria namely minimum charging tariff, shortest travelling distance and minimum waiting time at the charging station. This work also highlights the Big Data research thrusts, opportunities and challenges that are being evolved due to integration of cloud framework with the intelligent entities like smart meters, smart charging stations, xEVs etc. for commercial viability and implementation

\section{References}

[1] M.A.Hannan, F.A Azidin, A. Mohamed, "Hybrid electric Vehicles and their challenges”, Renewable and Sustainable Energy Reviews, Elsevier, vol. no. pp. 135-150, Sep-2013

[2] Yifeng He, Bala Venkatesh, Ling Guan, "Optimal Scheduling for Charging and Discharging of Electric Vehicles” IEEE Trans. Smart Grid, vol. 3, no. 3, pp.1095-1105, Sep-2012

[3] Victor del Razo, C Goebel, Hans-Arno Jacobsen, "Vehicle-Originating-Signals for Real-Time Charging Control of Electric Vehicle Fleets”, IEEE Trans. Transport Electrification, vol.1, no.2, pp-150-167, Aug-2015

[4] J Baek, Q Hieu Vu, J K Liu, X Huang, Y Xiang, "A Secure Cloud Computing Based framework for big data information management of Smart grids”, IEEE Trans. Cloud Computing, vol-3, no-2,pp-233-244, April/June-2015

[5] Melike Yigit, V. Cagri Gungor, Selcuk Baktir “Cloud Computing for Smart Grid applications”, Computer Networks, Elsevier, vol. 70, pp. 312-329, June-2014

[6] Ibrahim Abaker Targio Hashem, IbrarYaqoob, Nor Badrul Anuar, Salimah Mokhtar, Abdullah Gani, Samee Ullah Khan ,"The rise of "big data” on cloud computing: Review and open research issues”, Information Systems, Elsevier, vol. 47, pp. 98-115, Jan-2015.

[7] Samaresh Bera, Sudip Misra, Joel J. P. C. Rodrigues, "Cloud Computing Applications for Smart Grid: A Survey”, IEEE Trans. Parallel and Distributed Systems, vol. 26, no-5, pp-1477-1494, May-2015

[8] Panagiotis D. Diamantoulakis, Vasileios M. Kapinas, George K. Karagiannidis, "Big Data Analytics for Dynamic Energy Management in Smart Grids ”, Elsevier Big Data Research , vol. 2, no. 3, pp. 94-101, Sep. 2015

[9] M. Mureddu, A. Scala, A. Chessa, G. Caldarelli, M. Musio,A. Damiano, "An Agent Based Approach for the Development of EV fleet Charging Strategies in Smart Cities”, In Electric Vehicle Conference(IEVC), Florence, pp-1-8, 17-19 Dec,2014

[10] K Nandha Kumar, B Sivaneasan, P,Lam So “Impact of Priority Criteria on Electric Vehicle Charge Scheduling”, IEEE Trans. Transportation Electrification, vol. 1, no. 3, pp.200-210, Oct 2015

[11] Yifeng He, Bala Venkatesh, Ling Guan, "Optimal Scheduling for Charging and Discharging of Electric Vehicles" IEEE Trans. Smart Grid, vol. 3, no. 3, pp.1095-1105, Sep-2012 
[12] M.S Alam, "Key Barriers in the commercialization of Electric and plug-in Hybrid Electric Vehicles", Journal of Advances in Automobile Engineering, vol-2, no-2, 2013

[13] L. P. Fernández, T.G. San Román, R. Cossent, C. M. Domingo, and P.Frias, "Assessment of the impact of plug-in electric vehicles on Distribution Networks,” IEEE Trans. Power Syst., vol. 26, no. 1, pp. 206-213,Feb. 2011

[14] M. Kintner-Meyer, K. Schneider, and R. Pratt, "Impacts assessment of plug-in hybrid vehicles on electric utilities and regional U.S. power grids. Part 1: Technical analysis,” in Pacific NorthWest Nat. Lab., Richland, WA, USA, Nov. 2007.

[15] G. A. Putrus, P. Suwanapingkarl, D. Johnstion, E. C. Bentley, M. Narayana, "Impact of electric vehicles on power distribution networks”, In Proc. IEEE Vehicle Power and Propulsion Conf., Dearborn, MI, pp. 827-831, USA, 2009

[16] Hongyu Wu, M Shahidehpour, Ahmed Alabdulwahab, Abdullah Abusorrah "A Game Theoretic Approach to Risk Based Optimal Bidding Strategies for Electric Vehicle Aggregators in Electricity Markets With Variable Wind Energy Resources” IEEE Trans. Sustainable Energy, vol.7, no.1, pp-374-385, Jan-2016

[17] "SMART CHARGING: steering the charge, driving the change", A EURELECTRIC paper, March 2015

[18] M.S.Alam, "Vehicle to Cloud(V2C) remote management of Electric, Hybrid and plug-in Hybrid Electric Vehicle Charging", Patent Published in India, Patent Application \# 34590/DEL/2015

[19] US Department of energy, Office of Electricity Delivery and Energy Reliability ,Smart Grid Research \& Development, Multi-Year Program Plan-2010-2014

[20] K. Nandha Kumar B. Sivaneasan, P.H.Cheah, P. L. So, D. Z.W.Wang, "V2G Capacity Estimation Using Dynamic EV Scheduling” IEEE Trans. Smart Grid, vol-5, no-2, pp-1051-1060, March-2014

[21] Sergio Salinas, Ming Li, Pan Li,, Yong Fu, "Dynamic Energy Management for the Smart Grid With Distributed Energy Resources ”, IEEE Trans. Smart Grid, vol-4,no-4,pp-2139-2151 , Dec-2013

[22] V. del Razo, C. Goebel, and H.-A. Jacobsen, "Benchmarking a car originated- signal approach for real-time electric vehicle charging control” In Proc. IEEE Conf. PES Innov. Smart Grid Technol. (ISGT), Washington, DC, pp. 1-5, 19-22 Feb 2014

[23] Naouar Yaagoubi, Hussein T. Mouftah, "A Game Theoretic Approach for Plug-in Hybrid Electrical Vehicle Load Management in the Smart” in Electrical Power \& Energy Conference (EPEC), Halifax,NS, pp-1-6, 21-23 Aug 2013

[24] A. A. Eajal, M. F. Shaaban, E. F. El-Saadany, K. Ponnambalam "Fuzzy Logic-Based Charging Strategy for Electric Vehicles Plugged into a Smart Grid ”, in Smart Energy Grid Engineering (SEGE), Oshawa, ON, pp-1-6, 17-19 Aug 2015

[25] Huachun Han, Haiping Xu, Zengquan Yuan "Research of Interactive Charging Strategy for Electrical Vehicles in Smart Grids" In Electrical Machines and Systems (ICEMS), Beijing, pp.1-6, 20-23 Aug 2011

[26] Chenjie Maa, Juha Rautiainena, Dirk Dahlhausb, Akhilesh Lakshmana, J.-Christian Toebermanna and Martin Braun "Online optimal charging strategy for Electric Vehicles”, In 9th International Renewable Energy Storage Conference( IRES) , Energy Procedia, vol. 73 pp.173 - 181, 2015

[27] Jinbiao Xu and Vincent W.S. Wong, “An Approximate Dynamic Programming Approach for Coordinated Charging Control at Vehicle-to-Grid Aggregator”, Smart Grid Communications (SmartGridComm), Brussels, pp. 279-284, 17-20 Oct. 2011

[28] M. E. Khodayar, L. Wu, and M. Shahidehpour, "Hourly coordination of electric vehicle operation and volatile wind power generation in SCUC” IEEE Trans. Smart Grid, vol. 3, no. 3, pp. 1271-1279, Sep. 2012.

[29] Rani Yesudas, Roger Clarke, "Framework for Risk Analysis in Smart Grid Perspective Based Approach”, Proc. 8th Int'l Conf. on Critical Information Infrastructures Security (CRITIS 2013), Amsterdam, 16-18 September 2013. 